\title{
Evaluation of Reconfigurable Multiple and Compact Micro-Strip Antennas for MIMO Systems
}

\author{
B. Aslan, O. Dikmen, S. Kulaç and A. M. Elbir
}

\begin{abstract}
In wireless communication systems, the improvement of data rate and quality is a priority as a result of users' requests. During the improvement of these priority situations, there will also be a number of losses in the signals. It is known that the techniques used in large systems to solve such problems are not efficient in small systems like modem. Therefore, when using a smaller system such as a modem, the recommended techniques will have to be different. Multimode antenna designs will be an important alternative for next generation wireless communication systems especially for MIMO systems due to the physical advantages of small antenna structures. In this study, MIMO channel structure is shown and performance analysis and theories of compact micro-strip and reconfigurable multiple antenna designs used for the development of MIMO communication systems are mentioned.
\end{abstract}

Index Terms-Compact Multimode Micro-strip Antennas, MIMO, Reconfigurable Multiple Antenna Systems.

\section{INTRODUCTION}

$\mathrm{N}$ OWADAYS, great advances are observed with the developing technology in wireless communication field. At the same time, wireless communication is widely used in many industrial environments. The wireless communication system is simply an event that the signal is transmitted from the transmitter to the receiver. The signal between the transmitter and the receiver is transmitted to the user through source coding, channel coding, modulator, demodulator, channel decoding and source decoding [1]. In wireless communication systems, improving data rate and quality performances is a priority as a result of users' requests.

B. ASLAN, was Undergraduate with Department of Electrical Electronics Engineering University of Duzce University, Duzce, Turkey, (e-mail: aslan54batuhan@gmail.com)

O. DÍKMEN, is with Department of Electrical Electronics Engineering University of Duzce University, Duzce, Turkey, (e-mail: osmandikmen@duzce.edu.tr)

S. KULAÇ, is with Department of Electrical Electronics Engineering University of Duzce University, Duzce, Turkey, (e-mail: selmankulac@duzce.edu.tr)

A. M. ELBİR, is with Department of Electrical Electronics Engineering University of Duzce University, Duzce, Turkey, (e-mail: ahmetelbir@duzce.edu.tr)

Manuscript received September 13, 2017; accepted January 08, 2018. DOI: $10.17694 /$ bajece. 410247
The reason why data rate and quality cannot be sent at the desired level is the loss of some of the electromagnetic wave that travels in space. This loss situation is described as fading in the literature [2]. Basically, if we consider the transmission between a high-power base station and a mobile station, the elimination of the fading effect of such a system can be achieved by improving the low quality as a result of increasing the power of the base station [3]. However, if we consider this scenario for a modem, it is not possible to make such an analysis in terms of design, cost and electronic materials used for the modem [3]. Several suggestions have been put forward to remove this problem. In one of these proposals in the literature, MIMO systems were introduced. MIMO is basically a phenomenon in which information symbols to be sent are separated from symbols with more than one information without the need for high bandwidth. In this way, by transmitting these separated information symbols to multiple antennas, each symbol will be subjected to different fading, thus achieving gains in terms of efficiency and data rate. At the same time, the complexity of MIMO systems will be proportional to the number of used antennas [4]. Achieving gain such as an efficiency, data rate and low bandwidth is maintained with using variations dimensional process. These variations are achieved by modulation, signal processing and coding techniques [5]. In the literature, diversity is classified into 3 categories. These are divided into time diversity, frequency diversity and spatial diversity. In time diversity, there are divided information symbols. These divided information symbols provide benefits by experiencing fading at different time intervals. In frequency diversity, the available bandwidth is divided into segments and a gain is provided using a sign for each segment. In the spatial diversity, the gain is obtained by dividing the channel into multiple parallel channels by means of multiple antennas [5].

The rest of the paper is as follows: In the second part, MIMO channel structure is mentioned. In the third part, a brief introduction to the micro-strip antennas used in the Compact Multimode Micro-strip Antenna design has been given. In the fourth part, the MIMO antenna solution which can be restructured is mentioned. In the fifth section, the performance evaluation for used design of the Reconfigurable MIMO Antenna is given. In the sixth chapter, scenario of the Compact Multimode Micro-strip Antenna is mentioned theoretically. In the seventh section, a performance analysis of the solution 
design Compact Multimode Micro-strip Antenna is explained.

\section{MIMO CHANNEL STRUCTURE}

MIMO is a channel structure implemented to avoid the need for high gain and high bandwidth. What is important here is that the channel information must be known by the receiver in order for the transmitted information to reach the receiver [6].

Significant differences are observed when the channel capacity for MIMO systems, i.e., data rate, is compared to single-input single-output systems. With the assumption that different MIMO systems with single-input and single-output systems have the same channel losses and that the coding matrix is completely known to the receiver, it is apparent that the performance of MIMO systems is clearly increased in Fig. $1[6]$.

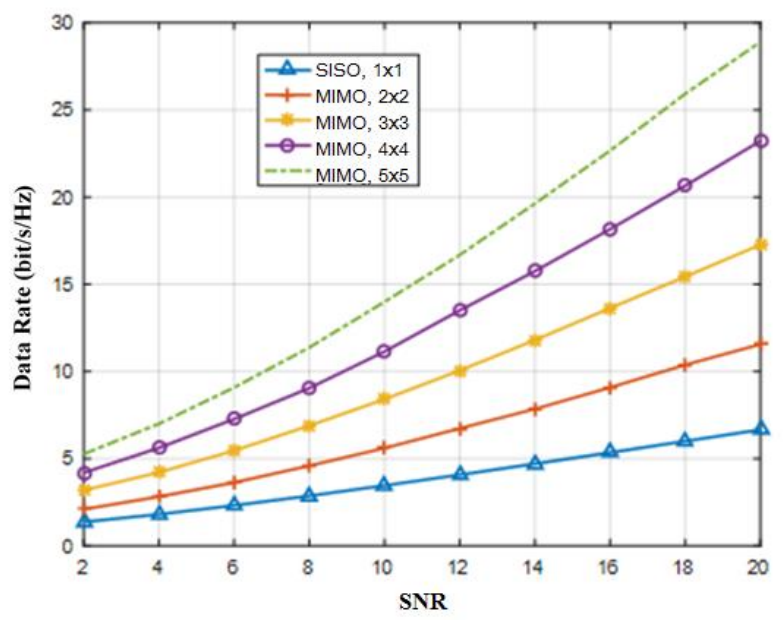

Fig.1. Data rate in MIMO Systems [6]

\section{MICRO - STRIP ANTENNAS}

In general, micro-strip antennas are the types of antennas that are important to gain the physical dimension. Having a low weight and flexible structure is a great physical advantage [3]. At the same time, having a shielded structure ensures that the deterioration of the assemblies is at a low level. In addition, the narrow frequency band, low output power, difficulty in formulating, and the delicate structure of power fluctuations are known as disadvantages. Fig. 2 is an example of a micro-strip antenna.

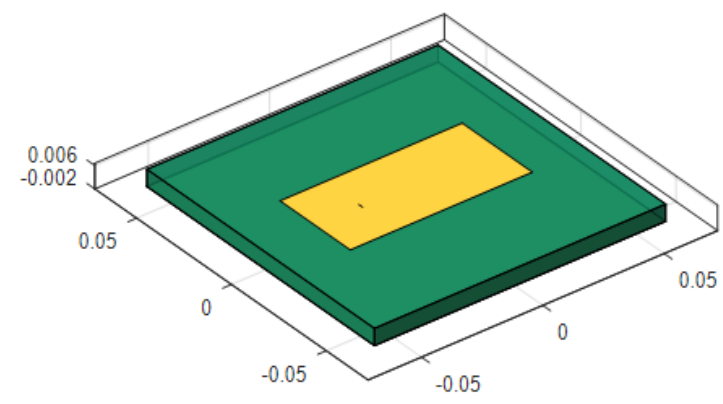

Fig.2. Rectangular micro-strip antenna

\section{SOLUTION OF RECONFIGURABLE MULTIPLE ANTENNA}

The radiation pattern obtained in antenna structures used in a standard MIMO system is always stable. The element factor of this pattern is shown as the constant $F(\alpha, \beta)$. Depending on the position of the receiver, the changing factor depending on various parameters such as the radiation angle is denoted as A $(\alpha, \beta)$. The product of these two constants pattern is the main pattern. For the $\mathrm{A}(\alpha, \beta)$ pattern, it is possible to improve with various coding techniques. For the $F(\alpha, \beta)$ element pattern, the reconfigurable antenna design is effective. For reconfigurable antenna design, antenna properties such as frequency, pattern, and polarization can be changed dynamically. It is possible to further increase this pattern gain with more than one reconfigurable MIMO antenna array. For a more detailed look at the system, there is a fixed transmitter with $\mathrm{M}$ antennas and a mobile receiver with $\mathrm{N}$ antennas. Equation (1) is used for the MIMO channel model. Here, the $\mathrm{H}$ channel matrix is used in determining the incoming signal. In this matrix, the channel gain coefficients change with the receiver's positional movements. In the MIMO channel structure, the matrix $\mathrm{X}$ comprises signal information and the information matrix is obtained from the $\mathrm{P}$ pre-coding matrix and the $\mathrm{S}$ vector set from the OFDM technique. We can express this with the following equation [7];

$$
\mathrm{X}=\mathrm{P}^{*} \mathrm{~S}
$$

The above-mentioned $\mathrm{A}(\alpha, \beta)$ pattern and the $\mathrm{P}$ pre-coding matrix can be obtained when properly selected according to the position of the receiver. This method is an application for classical MIMO systems. However, the reconfigurable antenna design has been introduced to change the elemental element $\mathrm{F}$ $(\alpha, \beta)$ factor. To this end, switching systems were created by adding diodes to the antenna design. Depending on the case where the pin diodes are conductive or insulator [5], appropriate modes are selected and gain is expected to be changed by changing the $F(\alpha, \beta)$ element factor. That is, the gain according to the position of the receiver will be obtained from both the factors of $F(\alpha, \beta)$ and $A(\alpha, \beta)$. The new gains will also affect the H-matrix due to the change in the pattern in the appropriate mode determined by the receiver's position. Previously, it has been mentioned that the matrix $X$ must be known in the receiver. Due to this reason, the matrix $H$ must also be known in the receiver. However, in order to select the appropriate mode and properly radiation pattern according to the $\mathrm{H}$ and $\mathrm{P}$ matrices in the transmitter, these matrix gains must also be known by the receiver. Therefore, this information is transferred to the receiver. In this design, which determines and matrices according to the mode of the appropriate $\mathrm{k}$ value for each position, three different mode suggestions have been made for the appropriate mode selection. The first one is the predictive mode. In this mode, the $\mathrm{H}$ and $\mathrm{P}$ matrices are reported to the receiver for all possible states, and the optimal mode is selected accordingly. According to this selection made new pattern is 
obtained. In this way, the gain is increased by changing the previously unchanged $F(\alpha, \beta)$ pattern. Second, smart mode. In this mode selection, instead of transmitting each of the $\mathrm{H}$ and $\mathrm{P}$ matrices that can be generated to the receiver, only the necessary matrices are transmitted. The third mode is fast mode. In this application, it is predicted that a quick selection should be made by creating a data for previously matched matrices [5].

\section{PERFORMANCE EVALUATION OF SOLUTION OF RECONFIGURABLE MULTIPLE ANTENNA}

Experimental results for the reconfigurable antenna structure are compared with the conventional omni antenna performance in Fig. 3 [5].

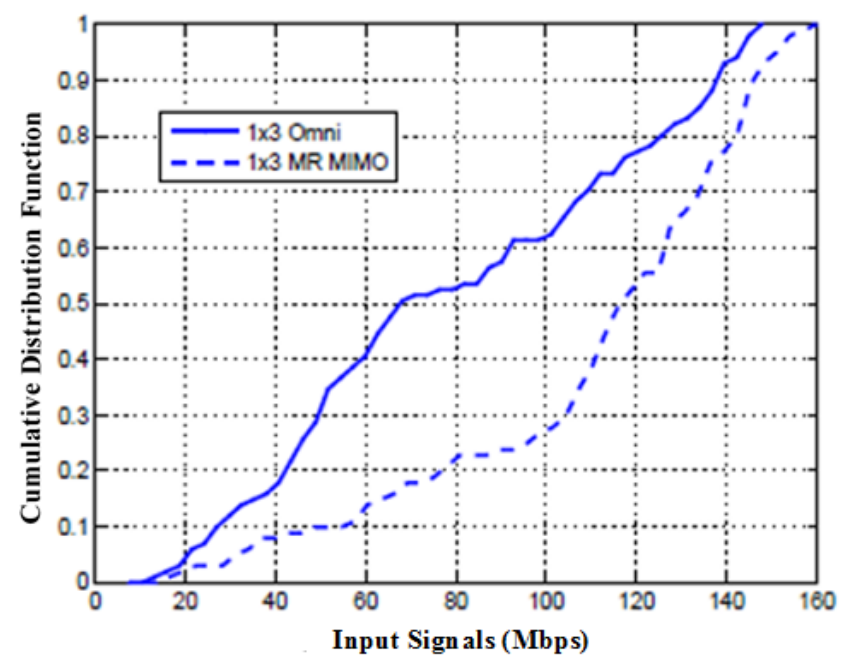

Fig.3. Corridor scenario- 1x3 SIMO [5]

Figure 1 shows the performance enhancement for the used reconfigurable antenna solution structure. However, studies done in the literature have some shortcomings as experimental and performance. In this system, it is seen that a performance improvement has been made in a reconfigurable MIMO antenna solution for systems that do not change the pattern factor of $F(\alpha, \beta)$ compared to a standard non-directional MIMO antenna, when examined on the basis of long distances. If we look at the system more objectively; this system is considered for short distances and the gain values are close to each other when the mode selection gain of the reconfigurable antenna design is compared with the fixed mode MIMO made with the standard directional antenna. Therefore, the situation where the performance gain of this system must be realized at suitable distances is a disadvantage. Besides, there is a mode selection with the assumption that only one user is in the experiments. In a multi-user case, the algorithms needed to select the appropriate mode are complex in the system. This is seen as one of the problems that arise in the development of the system. In cases where smart and fast modes are preferred, the most appropriate mode selection that should be selected introduces the use of intensive artificial intelligence. Here, the loss of the system load due to the increase of the system load at a certain distance based on the Path-Loss method is apparent from the study in Fig. 4 [6].

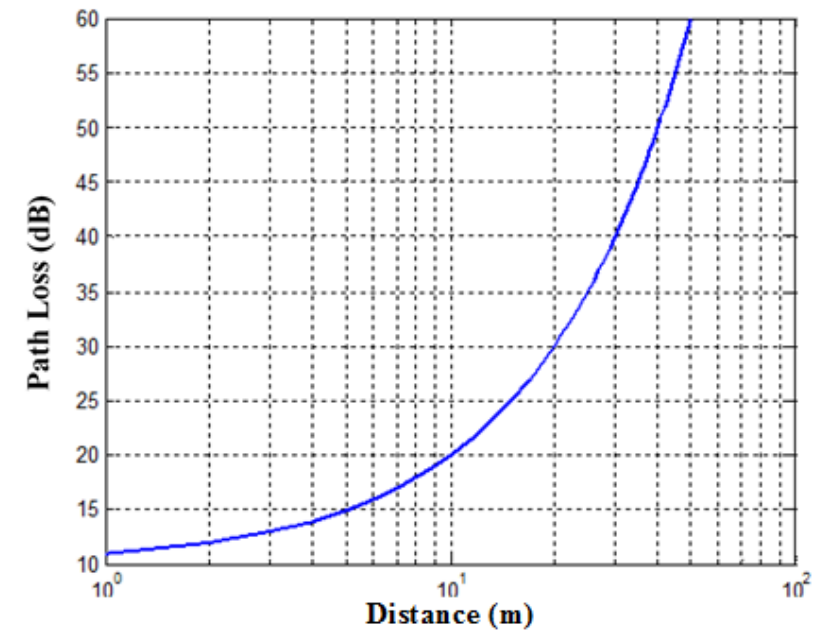

Fig.4. Power loss due to system software and hardware load [6]

In addition, there are some drawbacks to the mode selection operation determined by the position of the user. In the case of any artificial intelligence uncertainty, the pattern will not occur. Accordingly, breaks in the flow of data in correspondence will come into play. And at the same time, it is clear that there is no control of these situations during the system test. These are the disadvantages we have to consider.

\section{COMPACT MULTIMODE MICRO - STRIP ANTENNA}

In the literature, a different design is revealed by considering the advantages of micro-strip antennas. A design was created by placing two circular micro-strip antennas with two different modes on top of each other in a suitable position. This solution is a simple system that can be understood as a system because it provides pattern formation over two fixed modes unlike the reconfigurable antenna design. Previously, it was mentioned that micro-strip antennas have good screening and will not affect each other's patterns. However, according to the process performed in this design, since it is desired to obtain a new pattern through two different modes, when the angle of the antenna at the bottom is lower than the antenna at the top, the two antennas disturb each other's patterns. To prevent this, the antenna mode at the bottom is always higher than the antenna mode at the top. According to the design of this antenna, it is seen that performance improvement is achieved in experimental studies [5].

\section{PERFORMANCE ANALYSIS COMPACT MULTIMODE MICRO - STRIP ANTENNA}

The performance of the compact multi-mode micro-strip antenna is compared to the center feed (DP-ULA), dipole circular (CP-ULA) antennas using micro-strip antennas but with different sequences [7]. The compact multi-mode microstrip antenna is also called SCP-ULA. The power correlation between the used channel model and the antennas and the 
mode-dependent angle analysis are shown in Fig. 5 [7].

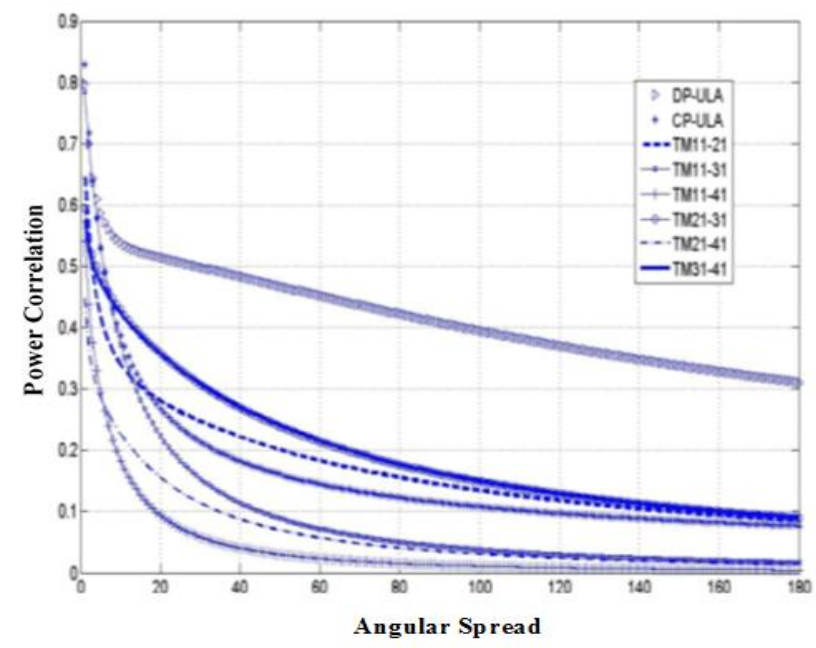

Fig.5. Antenna power correlation in different modes [7]

As can be seen from the graph in Figure 5, the compact micro-strip antenna solution (SCP-ULA) has a lower power correlation value than other antennas. A system with low power correlation has a higher spectral efficiency gain [8]. As we have already mentioned, and as can be seen from the analysis in Fig. 4 , the pattern at the top, where the angle of the antenna in the top of the antenna has risen, has higher power correlation and reduced spectral efficiency. The compact multimode microstrip antenna design, as it can be seen in the analysis, is a high performance alternative. In addition, it seems to be a great advantage for MIMO systems that need to be developed because of its low cost and design-enabling design. The high performance of the SCP-ULA design compared to other microstrip antenna designs makes it reasonable to develop over the SCP-ULA system. However, when considering a design to be done by adding one more layer to the SCP-ULA antennas with multi-layer design, the pattern angles brought by the modes lead to major distortions in the triple structure. As a result, SCPULA antennas are a constraint for such a development. We can explain this degradation from the incident radiation performance of the micro-strip antennas shown in Fig. 6.

As already mentioned, since micro-strip antennas have mixed mathematical pattern subtraction equations, it presents a challenge in determining the appropriate modes to be selected. But it is easier to solve these mixed problems with developing computer technology. Unlike the reconfigurable antenna design, this is one of the advantages of using fixed modes in this application, so that it does not need unnecessary algorithms and artificial intelligence applications. Since the feedback of the micro-strip antennas is very sensitive to the pattern distortion, it is necessary that a micro-strip antenna application with a multi-layer structure has a much more sensitive feedback system.

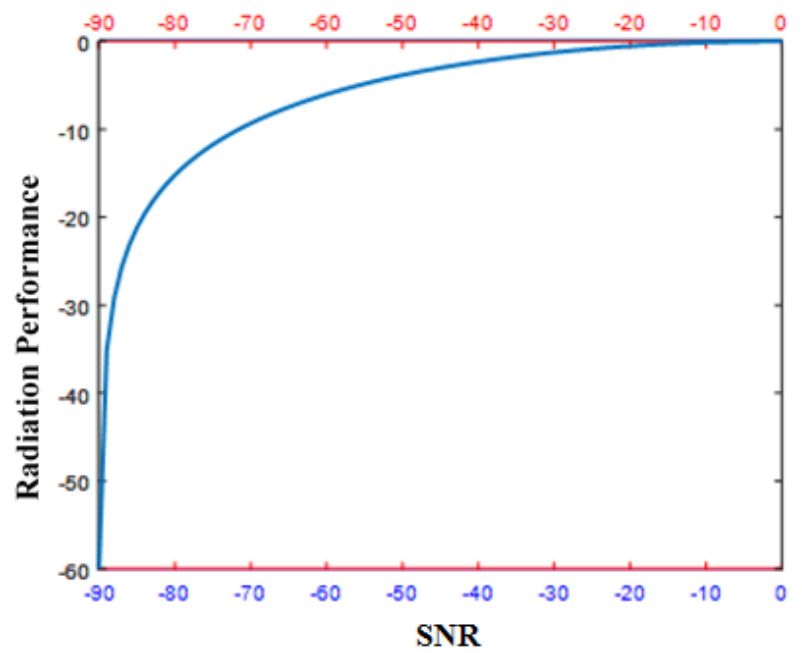

Fig.6. Performance of circular micro-strip antenna radiation-angle

\section{CONCLUSION}

In this study, antenna designs made for performance gain are investigated for two MIMO systems in the literature. These systems have been tried to be explained with the help of literature. At the same time, since the solution was recommended for the MIMO system, it is also mentioned about the MIMO channel structure and the necessity of MIMO systems. For better understanding of the systems, brief information is given about the types of antennas used in the designs. As a result, if the aim of this study is to be considered once more for the two systems, it has been tried to express the performance gains which are determined according to the position of the user as the basic logic and the optimum mode selection in the reconfigurable MIMO antenna design. It is understood that there are some disadvantages in these system tests, such as not testing the system in the case of multiple users and failing to obtain performance gains. Another solution proposal by the literature, the compact multimode micro-strip antenna, has been tried to specify the performance gains that can be achieved with a pattern that can be generated by a logical design in the case of fixed modes and fixed antenna types. In this paper, overall, the advantages that the antenna has brought are most efficiently utilized and the disadvantages are tried to be mentioned in the most objective manner.

\section{REFERENCES}

[1] M. Viswanathan, Simulation of Digital Communication Systems using Matlab, 2013, p.422.

[2] M. Viswanathan, On the Achievable Rate of Stationary Fading Channels, Springer, 2011, p.310.

[3] İ. Görgeç, Design of Dual-Mode Stacked Circular Microstrip Patch Antennas and Correlation / Spectral Efficiency Analysis for Mimo Configurations, Master Thesis, 2009, p.121.

[4] H. Zarrinkoub, Understanding LTE with Matlab, Wiley, 2014, p.508.

[5] E. Kurtulmaz, Çoklu Yeniden Ayarlabilir Çok Girisli Çok Çıkıslı Sistemlerde Dizayn ve Performans Analizi, Master Thesis, 2015, p.45.

[6] Y. S. Cho, J. Kim, W. Y. Yang, C.G. Kang, MIMO-OFDM Wireless Communications with MATLAB, Wiley, 2010, p.544

[7] A. Savaşçihabeş, Ö. Ertuğ, "IEEE 802.11n MIMO-OFDM WLAN haberleşme sistemlerinde kompakt multimod mikroşerit antenlerin korelasyon ve kapasite analizi", 3. Ulusal Haberleşme Teknolojileri ve Uygulamaları Sempozyumu, 2009, pp.127-132. 
[8] Ö. Çetin, B. Karakaya, H. A. Çırpan “3GPP LTE MIMO-OFDMA sistemlerde kanal kestirimi”, Signal Processing and Communications Applications Conference (SIU), April 2010, pp.129-132.

\section{BIOGRAPHIES}

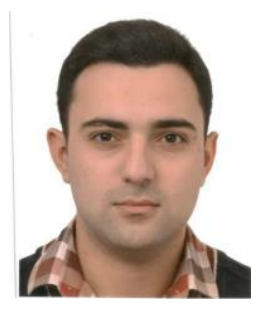

BATUHAN ASLAN was born in Sakarya, Turkey in 1994. He received his bachelor's degrees in electrical electronics engineering department at Duzce University, in 2017. His research interests are: Wireless Communication, Signal Processing.

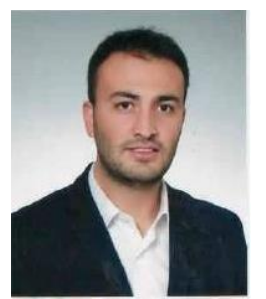

OSMAN DIKMEN was born in Yozgat, Turkey in 1989. He received his bachelors' degree in the Electronics Engineering Department at Gebze Technical University in 2013, master degree in the Electrical Electronics Engineering Department at Duzce University in 2017. And, he has been started $\mathrm{PhD}$ studying in the Electrical Electronics Engineering Department at Duzce University since 2017. He has been a research assistant in the Duzce University since 2014. His major areas of interests are: Wireless Communication, Signal Processing.

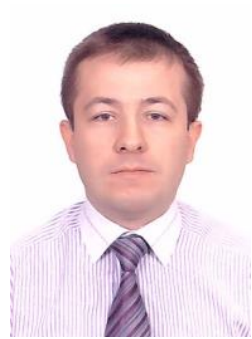

SELMAN KULAÇ was born in Düzce, Turkey. He received the B.S. degree from Ankara University in 2002 and M.S. degrees in electronics engineering from Ankara University, Turkey, in 2004 and the Ph.D. degree electronics engineering from Ankara University, Turkey, in 2012.

From 2002 to 2007, he was a Research Assistant with the Electronics Engineering Department, Ankara University. Since 2012, he has been an Assistant Professor with the Electrical Electronics Engineering Department, Duzce University. His research interests include telecommunication, wireless and mobile communication, cognitive radio, spectrum sensing, FPGA design, digital hardware design, video transmission, wireless implantable communication.

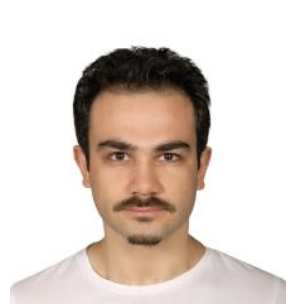

AHMET M. ELBIR was born in Malatya, Turkey in 1987. He received the B.S. degree from Firat University in 2009 and the Ph.D. from Middle East Technical University (METU) in 2016, both in electrical engineering. He is the recipient of 2016 METU best $\mathrm{Ph} . \mathrm{D}$ thesis award for his doctoral studies. Currently, he continues his studies at the Dept. of Electrical and Electronics Engineering, Duzce University, Turkey. His research interests include statistical signal processing, array signal processing, sparsity-driven convex optimization, and deep learning for radar applications. 